\title{
COMPETENCIA GLOBAL CHANGEMAKER Y AUTONOMÍA DE CENTRO. UN DESAFÍO POLÍTICO
}

\author{
$M^{a}$ Isabel Luis Rico \\ Tamara De La Torre Cruz \\ María Del Camino Escolar Llamazares \\ Esther Ruiz Palomo \\ Oiane Garbayo Maeztu \\ Universidad de Burgos \\ Diego Jiménez Palmero \\ Universidad de Sevilla
}

RESUMEN: El artículo expone los resultados de la investigación llevada a cabo para conocer los efectos sociopedagógicos y políticos derivados de la interacción entre la competencia changemaker y la autonomía de centro, abriendo una discusión sobre sus posibilidades y límites. Se parte del análisis de los argumentos que establecen una relación entre: a) el cambio competencial experimentado en el ámbito educativo para que los ciudadanos sean capaces de desenvolverse de forma satisfactoria y b) la autonomía de centro como rasgo organizativo catalizador de su implementación. Revisada la teoría de su interacción positiva, se comprueban sus efectos prácticos a través del análisis cualitativo de tres Proyectos Educativos de Centro de la Comunidad Autónoma de Castilla y León. En los proyectos se han desarrollado innovaciones relacionadas con la acción y el cambio social, implementando metodologías de Aprendizaje Servicio y de Comunidad de Aprendizaje y Enseñanza Democrática. Se identifican como categorías de análisis, la innovación, las competencias, la planificación de las actuaciones, los responsables y la evaluación. Los resultados muestran como la autonomía de centro, si bien de modo diferencial, permite en la práctica configurar los procesos de aprendizaje y la adquisición de la competencia global Changemaker.

PALABRAS CLAVE: competencia changemaker, autonomía de centro, proyecto educativo de centro, sostenibilidad, inclusión. 


\title{
GLOBAL CHANGEMAKER COMPETENCE AND CENTER AUTONOMY. A POLITICAL CHALLENGE
}

\begin{abstract}
This paper presents the results of an investigation carried out to study the socio-pedagogical and political effects, possibilities, and limitations derived from the interaction between changemaker competence and center autonomy. We build on the analysis of the arguments that establish a relationship between: a) the competence change experienced in the educational field so that citizens are able to function satisfactorily and b) center autonomy as an organizational feature activator of its implementation. Assuming the theory of positive interaction, its practical effects are verified through a qualitative analysis of three Educational Projects of the Autonomous Community Center of Castilla y León. In these projects, innovations related to action and social change have been developed, implementing Service Learning and Democratic Learning and Community Learning methodologies. We identify as categories of analysis, innovation, skills, planning of actions, the person in charge, and evaluation. The results show how the center autonomy, although in a differential way, allows in practice to configure the learning processes and the acquisition of the global Changemaker competence.
\end{abstract}

KEYWORDS: Changemaker competence, Center Autonomy, Center Educational Project, sustainability, inclusion.

Recibido: 09/12/2019

Aceptado: 27/12/2019

Correspondencia: $\mathrm{M}^{\mathrm{a}}$ Isabel Luis Rico. Universidad de Burgos, Facultad de Educación, C/Villadiego, 1, 09001 Burgos. Email: miluis@ubu.es.

\section{INTRODUCCIÓN}

Una de las improntas transversales que mejor caracterizan las sociedades modernas es el cambio acelerado de objetivos y procesos. Corriente generalizada que provoca controversia e incertidumbre en las instituciones encargadas de asegurar la prevalencia de la sociedad.

La educación, como una de las mediaciones sociales más universales para la transferencia crítica de normas y valores, vehicula no solo el aprendizaje de las competencias necesarias para un desenvolvimiento satisfactorio en la sociedad, sino también el aprendizaje de las habilidades individuales que permiten comprender los dinamismos del mundo actual. Igualmente, la educación permite construir entornos sostenibles y resilientes ante los cambios estructurales (Amadio, Opertti y Tedesco, 2014; Locatelli, 2018; Schleicher, 2018; Scott, 2015).

En estas circunstancias, nos asalta un interrogante en torno a qué educación en tiempos de hibridación proporciona una ventaja competitiva individual y hace pro- 
gresar la sociedad de modo sostenible. Desde distintas tribunas internacionales, justo es reconocerlo, se ha avanzado en principios y propuestas para una educación inclusiva, equitativa y de calidad, promoviendo oportunidades de aprendizaje permanente para todos, como parte fundamental de los Objetivos de Desarrollo Sostenible (ODS4) (Tawil, 2013; UNESCO, 2015a).

$\mathrm{Y}$, precisamente, en el núcleo mismo de esta educación y sociedad inclusivas, nos sorprende la pregunta de investigación sobre la que pretendemos profundizar con el ánimo de proporcionar criterios de política curricular ¿Qué competencias es necesario desarrollar para que los ciudadanos sean capaces de desenvolverse de forma autónoma y satisfactoria dentro de la sociedad actual y cuáles son sus condiciones de posibilidad?

\section{El DESCUBRIMIENTO DE LA COMPETENCIA GLOBAL CHANGEMAKER}

Conscientes del valor de la educación y de que los ciudadanos son el motor de la innovación y el cambio en las sociedades, organismos internacionales como la UNESCO y la OCDE, encontrando un amplio eco y respaldo por parte de la Unión Europea, han priorizado la disputada cuestión política de determinar cuáles han de ser las competencias de una nueva ciudadanía que exige un modelo social más equitativo y sostenible (UNESCO, 2009, 2015b, 2016).

La OCDE, fundamentalmente a través de los informes derivados de las evaluaciones internacionales PISA, aunque no como único instrumento de diagnóstico, ha identificado la necesidad de implementar una competencia que dote a los ciudadanos de la capacidad de apreciar y beneficiarse del ambiente complejo derivado de la interconexión, la diversidad y la volatilidad de los procesos sociales. Bajo la rúbrica Competencia Global se delimita un objetivo de aprendizaje multidimensional y permanente (OECD, 2018), que remite a una nueva función de los centros escolares como agentes responsables de facilitar a los estudiantes las experiencias y los espacios de aprendizaje:

"La capacidad de examinar cuestiones locales, globales e interculturales para comprender y apreciar las perspectivas y visiones del mundo de otras personas, participar en interacciones abiertas, adecuadas y efectivas con personas de diferentes culturas y actuar para el bienestar colectivo y el desarrollo sostenible." (OECD, 2018, p. 9)

El análisis pedagógico de la definición de Competencia Global permite establecer dimensiones bien diferenciadas, pero en estrecha conexión e interdependientes (véase Figura 1).

De forma complementaria, la Unión Europea dentro de su estrategia 2020 introduce la necesidad de desarrollar una educación que permite a los Estados miembros avanzar hacia sociedades con un crecimiento sostenible, integrador e inteligente (Comisión Europea, 2010, 2012), estableciendo cuáles han de ser las Competencias Clave para conseguir una educación, formación y aprendizaje permanente inclusivo y de calidad que permita asegurar la resiliencia y 
la capacidad de adaptación al cambio de los ciudadanos (Consejo Europeo, 2018). El resultado, aunque heterogéneo y no exento de crítica, propicia los ejes fundamentales y estimula la investigación pedagógica sobre estos ámbitos: Competencia en lectoescritura; Competencia multilingüe; Competencia matemática y Competencia en ciencia, tecnología e ingeniería; Competencia digital; Competencia personal, social y de aprender a aprender; Competencia ciudadana; Competencia emprendedora; Competencia en conciencia y expresión culturales. Resulta relevante subrayar que en esta misma recomendación establece que son las competencias emprendedoras, sociales y cívicas las que cobran más importancia para poder alcanzar la promoción de los valores comunes y la construcción de la dimensión europea de ciudadanía (Comisión Europea, 2018).

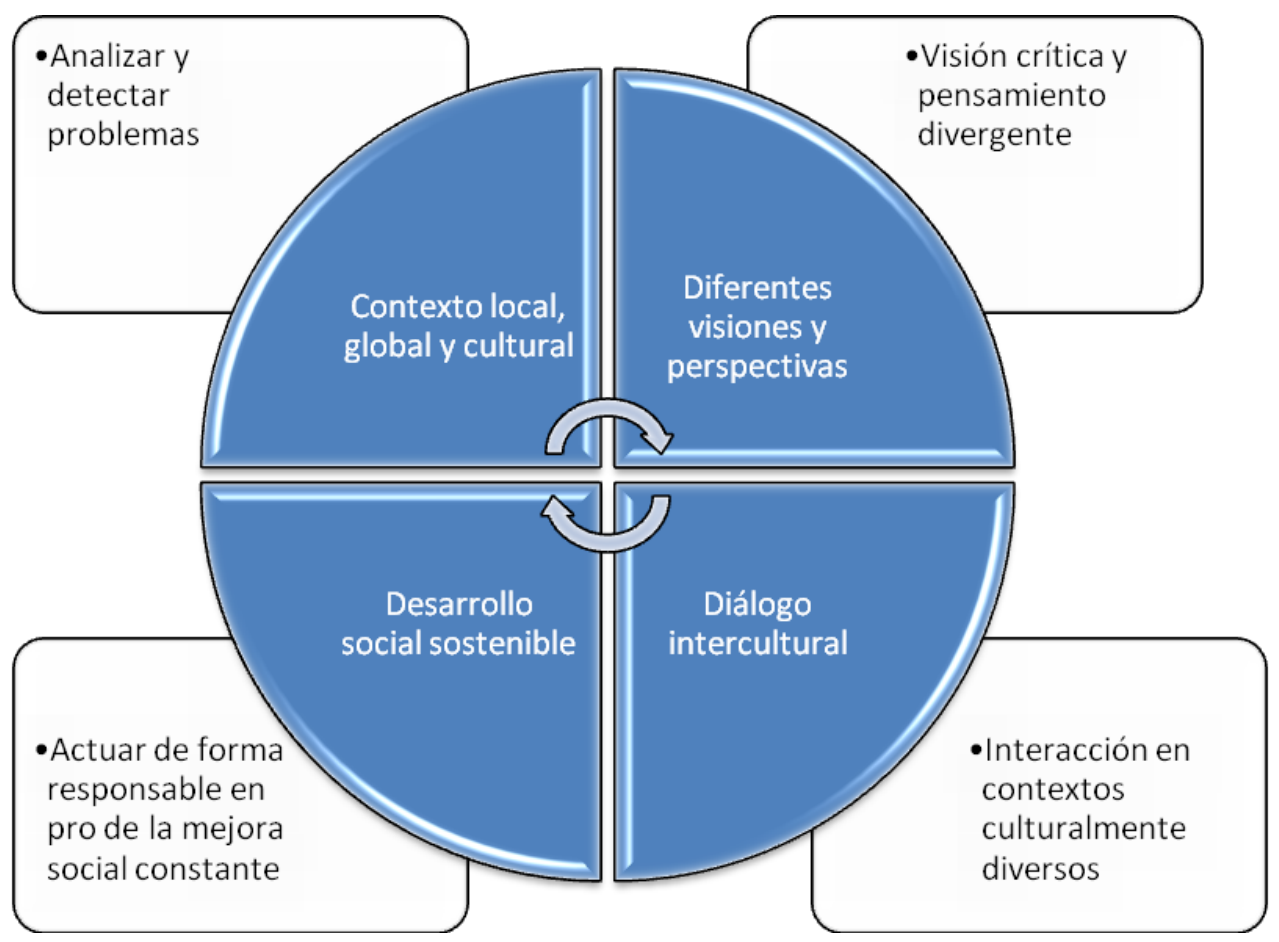

Figura 1. Dimensiones de la Competencia Global (OECD, 2018)

Si consideramos conjuntamente las dimensiones establecidas dentro de la Competencia Global y las competencias que desde la UE se señalan como necesarias para la construcción de la ciudadanía europea, percibimos la urgente necesidad de que las instituciones educativas, revisando su funcionalidad, instrumenten una respuesta estratégica ordenada al desarrollo de conocimientos, habilidades, actitudes y valores necesarios para promover cambios sociales que permitan la construcción de una ciudadanía inclusiva, sostenible, innovadora y resiliente. Un ambicioso cambio en los rasgos, no solo estructurales, sino también funcionales, de las instituciones edu- 
cativas que nos conducen al ámbito del emprendimiento social considerado como el proceso y oportunidad de crear valor para la sociedad a través del cambio y la innovación con un impacto en la comunidad (Bravo Monge, 2016; Fernández-Laviada, 2015). En este eje de coordenadas, se comprende y cobra sentido nuestro planteamiento de la Competencia Global Changemaker como síntesis de la competencia global propuesta por la OCDE y las competencias emprendedora, social y cívica de las competencias clave de la UE.

En efecto, la Competencia Global Changemaker, además de una reconsideración de los fines de la educación, también promueve un cambio profundo en las escuelas, excediendo la simple adquisición de conocimientos, hacia la innovación, la resolución de problemas, la colaboración, con el fin de promover el cambio en los actuales contextos interculturales. De tal forma que el ciudadano se convierta en un agente social de cambio, capaz de detectar y atenuar los procesos perniciosos de la sociedad. Además, su dimensión social y cívica proporciona el conocimiento del entorno, de las relaciones que se establecen como ciudadanos, de los valores democráticos, del contexto, incluyendo los aspectos de participación, cambio, sostenibilidad e innovación. Y en esta línea, la competencia emprendedora aporta los conocimientos para acometer la acción y aspectos tan relevantes para el desarrollo individual como es la resiliencia, ineludible en la sociedad actual (Azqueta Día de Aldaá, 2019).

Ahora bien, la recualificación internacional del cambio competencial ha suscitado una reflexión en torno a los cambios pedagógicos idóneos para promover estas nuevas competencias, abriendo flancos críticos que reubican al estudiante, una vez más, en el centro de un aprendizaje autónomo y crítico. Parece probado que la competencia global changemaker se relaciona con las destrezas meta-cognitivas, ya que permite relacionar lo aprendido con los conocimientos previos a través de su aplicación en la realidad con el objetivo de cambiarla y mejorarla. Consecuentemente, cobran fecundidad heurística y metodológica las pedagogías basadas en el aprendizaje colaborativo, aprendizaje basado en proyectos, aprendizaje cooperativo, aprendizaje basado en problemas, desing thinking, aprendizaje basado en el pensamiento o thinking based learning, aprendizaje por descubrimiento, aprendizaje servicio, aprendizaje por construcción o maker, entre otras, que potencian que los estudiante transfieran sus conocimientos de forma participativa, siendo capaces de aplicar los aprendizajes a diferentes situaciones y contextos (Montanero Fernández, 2019; Scott, 2015).

No solo se trata de algo posible, sino de algo urgente. Nos referimos a la racionalidad instrumental, crítica y creativa de la política educativa para afrontar el reto de hacer converger los cambios teleológicos derivados de la instalación educativa de la competencia changemaker con las reformas curriculares y metodológicas que deben operarse en los centros educativos (Viennet y Pont, 2017). Interacción que nos conduce a una consideración complementaria en torno a la autonomía de centro como elemento catalizador del desarrollo de innovaciones metodológicas en los sistemas escolares. 


\section{LA AUTONOMÍA DE CENTRO COMO OPORTUNIDAD DE INNOVACIÓN}

Los sistemas educativos actuales han heredado culturas organizativas y estructuras de poder procedentes de épocas industriales, donde la homogenización era una de las prioridades. En la actualidad, se detecta una mayor sensibilidad e interés creciente sobre las contribuciones de la política educativa a la eficiencia de los sistemas educativos, cuyas prioridades se concentran en torno a la división de responsabilidades o gobernanza; la mejora de los resultados, las habilidades y la formación del profesorado y la definición de prioridades y objetivos educativos nacionales.

En este escenario, no resulta exagerado afirmar que la gobernanza emerge como una línea medular en el cumplimiento de los objetivos de los sistemas educativos, pudiendo distinguirse desde 2008 a 2019 hasta cinco prioridades políticas en este área: determinar la distribución de responsabilidades en el sistema; la definición de objetivos educativos en el ámbito nacional; involucrar a los agentes educativos en la toma de decisiones; implementar mecanismos de garantía de la calidad y fortalecer los datos para efectuar evaluaciones de calidad y permitir la rendición de cuentas (OECD, 2019b).

En lo que respecta, de forma directa, a la distribución de responsabilidades, la OECD señala como principio de acción la dotación de mayor autonomía a los centros junto con la determinación de las responsabilidades en la toma de decisiones. Los resultados de las evaluaciones PISA advierten que los países donde los centros educativos tienen una mayor autonomía respecto a los temas propios de enseñanza obtienen mejores rendimientos. La autonomía de centro debe de tener de forma previa una definición de expectativas claras y objetivos educativos, para permitir que la concreción se realice en función de las características propias del contexto para satisfacer los objetivos marcados, centrándose en la innovación. De otra forma, una autonomía sin objetivos provocaría aislamiento educativo y el desequilibrio en cuanto al rendimiento (OECD, 2019a).

En España, los informes de evaluación indican que la autonomía de centro es una de las áreas en las que se ha de seguir trabajando dentro de las políticas educativas para mejorar los resultados de las evaluaciones (Calleja, Collado, Macías y San José, 2012). A pesar de la existencia de una normativa legal proclive a la autonomía, su eficacia no traspasa el ámbito formal por cuanto todavía no se ve reflejada en las evaluaciones internacionales.

La legislación educativa establece tres áreas susceptibles de autonomía en los centros educativos: Autonomía pedagógica, Autonomía de organización y funcionamiento y Autonomía de gestión. Nuestro interés investigador se centra en el desarrollo de la autonomía pedagógica, ya que es donde se concreta la libertad de adaptación de los procesos pedagógicos para que el alumnado desarrolle las nuevas competencias, incluyendo aquí las innovaciones en el ámbito de las metodologías.

El documento público en el que se plasma la autonomía de los centros es el Proyecto Educativo de Centro (PEC), objeto de regulación diferenciada por parte de las 
legislaciones autonómicas en cuanto a sus elementos, carácter, finalidad y procedimiento de aprobación (Sancho, 2014).

\section{Metodología de LA inVestigación}

Nuestro objetivo general de investigación se centra en determinar las condiciones para implantar acciones de innovación metodológica y curricular en los centros escolares, a través del ejercicio de su autonomía pedagógica y organizativa para llegar a desarrollar la competencia global changemaker.

En función del objetivo general planteado nuestra investigación plantea el siguiente objetivo específico: Analizar Proyectos Educativos de Centro donde se desarrollen acciones de innovación metodológicas relacionadas con la competencia changemaker.

Para dar satisfacción a los objetivos planteados centramos nuestro análisis en tres Proyectos Educativos de Centro de la Comunidad Autónoma de Castilla y León, donde hemos desarrollado innovaciones relacionadas con la acción y el cambio social, a través de metodologías de Aprendizaje Servicio (ApS), y de Comunidad de Aprendizaje y Enseñanza Democrática.

Se trata de un estudio cualitativo que sigue la teoría fundamentada (Mella, 2003; Strauss y Corbin, 2002), teniendo como propósito central elaborar una teoría construida a partir de los datos recopilados, analizados de forma sistemática y categorizados con el objeto de hallar las relaciones entre los diversos conceptos desprendidos a partir de los mismos datos, en este caso, los documentos de autonomía pedagógica de centros donde se desarrollan proyectos innovadores relacionados con la acción y el cambio social, obteniendo una mejor comprensión de la realidad estudiada.

Así la teoría fundamentada posee como característica central la fundamentación de los conceptos en los datos (Strauss y Corbin, 2002: 14), e incorpora, adicionalmente el componente de creatividad por parte del investigador, permitiendo a través del análisis la construcción de la teoría que subyace.

La selección de los centros se ha realizado en base a los criterios de: a) realización de proyectos de innovación dentro del centro escolar con más de 2 cursos de implantación; b) pertenencia a la misma comunidad autónoma para asegurar la misma legislación en materia de educación y de autonomía de centros; y c) disponibilidad para el envío de los PEC. Las características de los centros seleccionados se muestran en la Tabla 1.

Tabla 1. Características de los centros educativos seleccionados

\begin{tabular}{|c|c|c|c|}
\hline CENTRO & ÁMBITO & COMUNIDAD & PROYECTO DE INNOVACIÓN \\
\hline Centro 1 & Rural & Castilla y León & Comunidad de Aprendizaje \\
\hline Centro 2 & Rural & Castilla y León & Aprendizaje Servicio \\
\hline Centro 3 & Rural & Castilla y León & Proyecto Roma (Educación Democrática) \\
\hline
\end{tabular}


Para desarrollar el análisis de contenido de los documentos seleccionados se ha utilizado el software Atlas. Ti v.8 identificando, de forma general, diferentes categorías de análisis. Esto ha permitido establecer la presencia dentro del PEC de las actuaciones de innovación que desarrolla el centro analizado. Las categorías analizadas y sus dimensiones se pueden observar en la Tabla 2.

Tabla 2. Categorías y dimensiones de análisis

\begin{tabular}{|c|c|c|}
\hline DIMENSIÓN & CATEGORÍA & DELIMITACIÓN DE LA CATEGORÍA \\
\hline Innovación & Definición de la innovación & $\begin{array}{l}\text { Dentro del PEC se encuentra reflejada la } \\
\text { conceptualización de la innovación que } \\
\text { se desarrolla en el centro. }\end{array}$ \\
\hline Competencia & $\begin{array}{l}\text { Competencias con las que } \\
\text { se relaciona }\end{array}$ & $\begin{array}{l}\text { El desarrollo de la acción innovadora se } \\
\text { relaciona con las competencias que han } \\
\text { de alcanzarse dentro de las etapas edu- } \\
\text { cativas en las que se enmarca. }\end{array}$ \\
\hline \multirow{3}{*}{ Organización } & $\begin{array}{l}\text { Planificación de } \\
\text { actuaciones }\end{array}$ & $\begin{array}{l}\text { El proyecto de innovación se encuentra } \\
\text { desglosado en las acciones que se han de } \\
\text { desarrollar para llevarlo a cabo durante } \\
\text { el periodo escolar. }\end{array}$ \\
\hline & $\begin{array}{l}\text { Responsables de la } \\
\text { actuación }\end{array}$ & $\begin{array}{l}\text { En el PEC se refleja los docentes respon- } \\
\text { sables del proyecto de innovación, así } \\
\text { como las etapas en las que se ha de im- } \\
\text { plementar y los docentes implicados. }\end{array}$ \\
\hline & $\begin{array}{l}\text { Resultados alcanzados/ } \\
\text { Evaluación }\end{array}$ & $\begin{array}{l}\text { Se muestra las evaluaciones previas del } \\
\text { proyecto sobre el que se asienta su de- } \\
\text { sarrollo, así como los procedimientos de } \\
\text { evaluación en relación a las competen- } \\
\text { cias marcadas. }\end{array}$ \\
\hline
\end{tabular}

\section{Resultados}

Los resultados alcanzados por categorías según los datos analizados de cada centro se recogen en la Tabla 3.

Como se puede observar en la Tabla 3 en el centro 1, basado en la comunidad de aprendizaje, no hay una definición explícita del proyecto que se desarrolla dentro del mismo, explicando la relación con el contexto como un conocimiento, sin hacer hincapié en la acción que conlleva la participación de la comunidad educativa en todos los ámbitos del proceso escolar. EL centro 2, basado en el Aprendizaje Servicio, establece de forma clara la definición de lo que entiende que ha de ser un proyecto de innovación basado en esta metodología. En el centro 3, que desarrolla el proyecto Roma como metodología democrática, establece desde la introducción que todos los elementos del PEC se han de centrar en el desarrollo del mismo. 
Tabla 3. Resultados en la categoría sobre definición de la innovación

\begin{tabular}{|c|c|}
\hline CENTRO 1 & $\begin{array}{l}\text { En el Proyecto Educativo del centro } 1 \text { no hay referencia a la implantación del pro- } \\
\text { yecto de Comunidad de Aprendizaje, no obstante, en diferentes apartados del PEC } \\
\text { se realizan alusiones a la necesidad de que la comunidad educativa esté presente } \\
\text { en diversos ámbitos de la organización del centro. Las referencias encontradas son: } \\
\text { “...También desde la comunidad educativa se potenciará y prestará colaboración } \\
\text { a las actividades socioculturales que se programen y desarrollen en el entorno del } \\
\text { centro..." (en la organización general de centro) } \\
\text { “... Realización de actividades en las que estén implicados todos los integrantes de } \\
\text { la comunidad escolar. } \\
\text {. Realización de unas Jornadas Culturales. } \\
\text {. Invitar a los padres a que participen en alguna actividad de aula...” (En los obje- } \\
\text { tivos de convivencia) } \\
\text { “...Elaborar y distribuir por la Comunidad Educativa información sobre el centro } \\
\text { (servicios educativos, instalaciones, Proyecto Educativo, equipo de profesores) ..." } \\
\text { (En los objetivos de convivencia) } \\
\text { “...El colegio procurará participar en aquellas actividades promovidas por estos } \\
\text { organismos cuando se prevea que su puesta en práctica va a redundar en beneficio } \\
\text { de la comunidad educativa..." (Funciones de la dirección) } \\
\text { “...Se colaborará con las entidades locales en aquellas actividades organizadas... } \\
\text {...El centro colaborará en la medida de sus posibilidades con asociaciones huma- } \\
\text { nitarias... } \\
\text {... Las actividades programadas en función de la cooperación que se establezcan } \\
\text { han de ser de carácter educativo y estar recogidas en la PGA. En el caso de que no } \\
\text { sea así habrá de valorarse si su esporádica inclusión en la programación no perjudi- } \\
\text { ca a la organización escolar..." (Plan de atención a la diversidad) }\end{array}$ \\
\hline CENTRO 2 & $\begin{array}{l}\text { En este centro la definición del proyecto de ApS se encuentra dentro del proyecto } \\
\text { de autonomía en los planes educativos. } \\
\text { “...El aprendizaje-servicio, el alumnado identifica en su entorno próximo una si- } \\
\text { tuación con cuya mejora se compromete, desarrollando un proyecto solidario que } \\
\text { pone en juego conocimientos, habilidades, actitudes y valores. } \\
\text { Es una práctica educativa en la que alumnos y alumnas aprenden mientras actúan } \\
\text { sobre necesidades reales de su comunidad y entorno con la finalidad de mejorar- } \\
\text { lo..." (Planes educativos) }\end{array}$ \\
\hline CENTRO 3 & $\begin{array}{l}\text { En el Centro se presenta el proyecto de innovación, Proyecto Roma o de educación } \\
\text { democrática, desde la introducción del Proyecto Educativo de Centro, estando pre- } \\
\text { sente sus referencias al Proyecto Roma en todos los elementos que componen el } \\
\text { PEC, ya que lo incluyen como modelo educativo para todo el centro. } \\
\text { "...Ese modelo educativo está sustentado en los principios del Proyecto Roma y es } \\
\text { el que impregna el Proyecto Educativo del Centro..." (Introducción) }\end{array}$ \\
\hline
\end{tabular}

En la Tabla 4 vemos como en el centro 1 no hay una referencia explícita a la relación que se establece entre el desarrollo del proyecto de comunidad de aprendizaje con las competencias que han de alcanzar los alumnos ni con los objetivos. En el caso del centro 2 se relaciona con las competencias del ámbito social y con el desa- 
rrollo de una ciudadanía activa y responsable. Igualmente, se señala su aportación a la configuración de los valores y actitudes prosociales, así como las habilidades para la vida, siendo también un aspecto necesario para trasladar los aprendizajes de los alumnos a la vida y a su entorno. Dentro del centro 2 se hace referencia a la necesidad de desarrollar el espíritu emprendedor como parte fundamental del desarrollo competencial, dentro de la conceptualización del emprendimiento social, como vía para transformar la realidad social y mejorarla.

Tabla 4. Resultados en la categoría sobre las competencias con las que se relaciona

\begin{tabular}{|c|c|}
\hline CENTRO 1 & No se relaciona con las competencias de forma explícita \\
\hline CENTRO 2 & 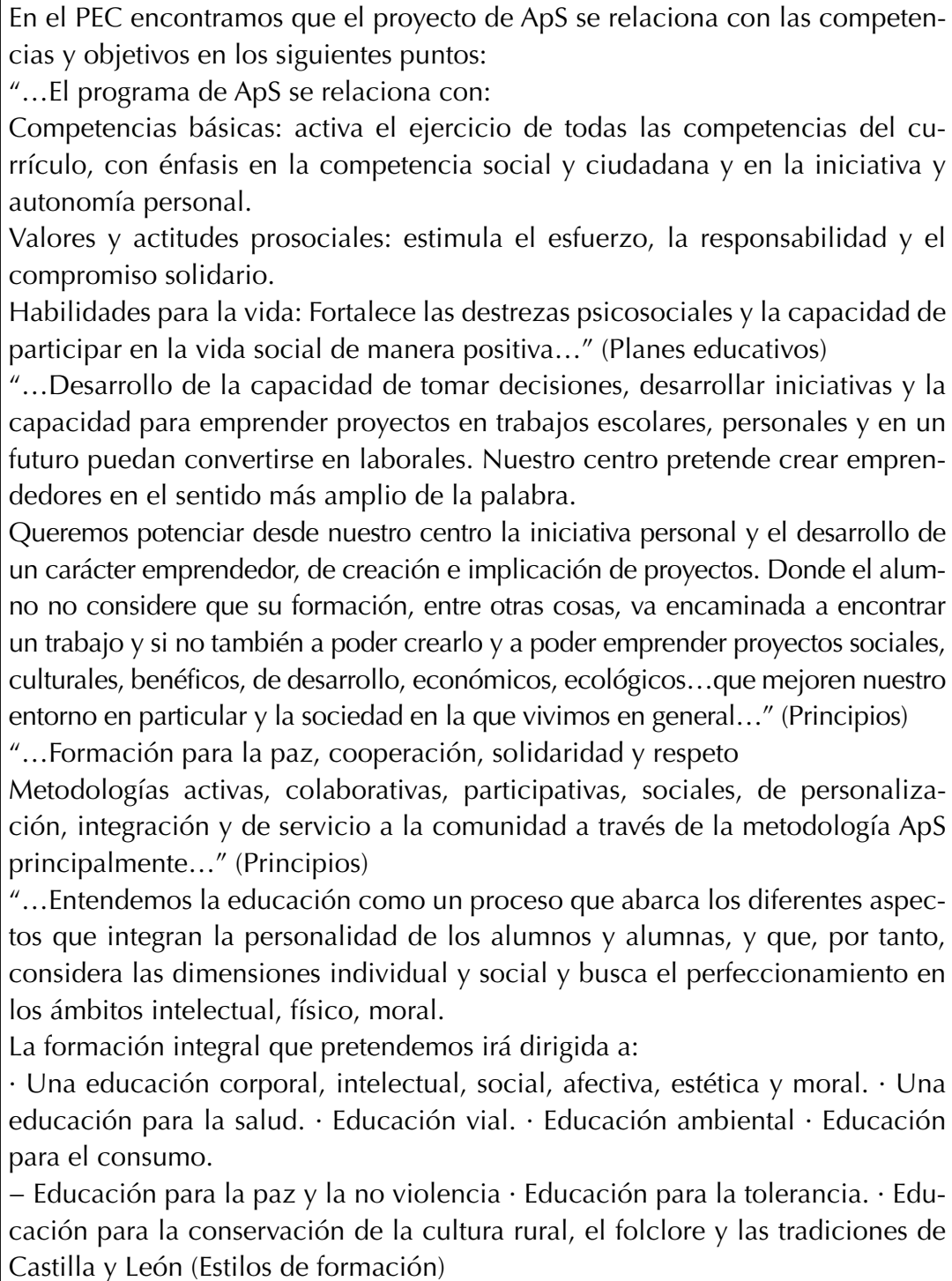 \\
\hline
\end{tabular}




\begin{tabular}{|c|c|}
\hline & $\begin{array}{l}\text { "...Se da especial importancia al desarrollo de las competencias en los } \\
\text { alumnos para que los aprendizajes adquiridos en esta etapa puedan verse } \\
\text { trasladados a la vida real y a su participación activa en la misma..." (Dimen- } \\
\text { siones educables) } \\
\text { "...El Centro intentará desarrollar el espíritu crítico, la capacidad de dis- } \\
\text { cusión y de decisión y una actitud participativa, dentro de la diversidad de } \\
\text { ritmos evolutivos y de capacidades intelectuales, dando importancia tanto a } \\
\text { la formación humana como a la adquisición de técnicas, contenidos básicos } \\
\text { y competencias..." (Líneas metodológicas) }\end{array}$ \\
\hline & $\begin{array}{l}\text { "...La preparación para participar activamente en la vida social y cultural. } \\
\text { Interviniendo en el medio y tomando una actitud participativa..." (Fines edu- } \\
\text { cativos) } \\
\text { "...Continuar realizando proyectos ApS. a nivel de centro para trabajar las } \\
\text { diferentes necesidades sociales que se detecten, así como los ODS, con el } \\
\text { apoyo de las entidades implicadas en cada proyecto, a la vez que continua- } \\
\text { mos mejorando los proyectos ya realizados como la ruta de NATURPUE- } \\
\text { BLO..." (Objetivos generales del centro) }\end{array}$ \\
\hline CENT & $\begin{array}{l}\text { Se relaciona con las competencias y objetivos en los siguientes puntos del PEC: } \\
\text { "... Una comunidad de convivencia y aprendizajes que forme a una ciuda- } \\
\text { danía culta, librepensadora, dialogante, responsable, autónoma y feliz..." } \\
\text { (Pretensiones) } \\
\text { "...El centro y el aula como una comunidad de convivencia y aprendi- } \\
\text { zajes... Construcción social del conocimiento..." (Proyectos de Investiga- } \\
\text { ción). } \\
\text { “...Construcción de la democracia. Participación de las familias del cen- } \\
\text { tro..." (Principios) } \\
\text { "... Hacer de nuestro centro una comunidad de convivencia y aprendizajes } \\
\text { entre familias y profesorado, una propuesta educativa basada en la partici- } \\
\text { pación y en el diálogo..." (Objetivos generales del centro) } \\
\text { “...Tanto en la clase de infantil como en primaria se organiza el currículum } \\
\text { según el modelo educativo del Proyecto Roma y hacemos a través de proyec- } \\
\text { tos de investigación y los definimos como un modo de aprender a aprender } \\
\text { en cooperación..." (Metodología) }\end{array}$ \\
\hline
\end{tabular}

En el centro 3 forma parte del proyecto educativo de forma amplia por lo que está presente en las pretensiones educativas del centro, dentro de las características de una sociedad democrática, como ciudadanía libre, dialogante y responsable. En este sentido establece el aula como unidad de actuación en la que el desarrollo de las competencias democráticas, pueden adquirirse a través de la experiencia (véase Tabla 4).

En el centro 1 no se recoge en el PEC la planificación de actividades que desarroIlen el proyecto, mientras que en los centros 2 y 3 hay un desarrollo pormenorizado de las fases que ha de recorrer el docente para llevar a cabo el proyecto, tanto el de ApS como el Proyecto Roma (véase Tabla 5). 
Tabla 5. Resultados en la categoría sobre la planificación de las actuaciones

\begin{tabular}{|c|c|}
\hline CENTRO 1 & $\begin{array}{l}\text { No se encuentran referencias específicas a las actuaciones que se desarrollan en } \\
\text { el ámbito del proyecto de comunidad de aprendizaje }\end{array}$ \\
\hline CENTRO 2 & $\begin{array}{l}\text { El proyecto de innovación de ApS se encuentra planificado con las siguientes } \\
\text { etapas: } \\
\text { “...Sus fases para nuestros proyectos son las siguientes: } \\
\text { Preparación del educador: Análisis del grupo y de cada participante; Detección } \\
\text { de necesidades, servicios y transiciones; Vinculación curricular; Planificación } \\
\text { del proyecto. } \\
\text { Planificación con el grupo: Motivación; Diagnóstico del entorno y definición } \\
\text { del proyecto; Organización del trabajo; Reflexión sobre aprendizajes de la pla- } \\
\text { nificación. } \\
\text { Ejecución con el grupo: Actividad; Cursos; Desarrollo } \\
\text { Evaluación del grupo y del educador..." (Planes de centro) }\end{array}$ \\
\hline CENTRO 3 & $\begin{array}{l}\text { EL desarrollo del Proyecto Roma se centra en los siguientes aspectos: } \\
\text { “...Iniciamos el proceso a través de lo que se conoce en el Proyecto Roma como } \\
\text { Cuestiones Previas } \\
\text { 1. Las clases comienzan conociéndonos a través de la conversación y el diálo- } \\
\text { go. Y, en ese conversar, las niñas y niños empiezan a aprender que todo el } \\
\text { mundo en clase puede aprenderlo todo si se ayudan entre sí, que la mejor } \\
\text { forma de aprender es ayudar a aprender, y que cuando alguien tiene difi- } \\
\text { cultades para aprender algo, va a aprenderlo si el resto le ayuda (aprendizaje } \\
\text { cooperativo). } \\
\text { 2. Se consensuan las normas de convivencia -de clase, asamblea y grupo-, te- } \\
\text { niendo consciencia de los valores que salvaguardan y los posibles perjuicios } \\
\text { de su incumplimiento. } \\
\text { 3. Consideramos el conocimiento como una construcción social, por tanto, } \\
\text { trabajamos en grupo, y de ahí emerge la necesidad de asumir cuatro respon- } \\
\text { sabilidades: coordinador o coordinadora, portavoz, secretaria o secretario y } \\
\text { responsable de material. } \\
\text { 4. Aprendemos después que la clase es como un cerebro, con cuatro Zonas de } \\
\text { Desarrollo y Aprendizaje: Zona de Procesos Cognitivos y Metacognitivos, del } \\
\text { Lenguaje, de la Afectividad y de la Autonomía. } \\
\text { En consecuencia, tenemos que buscar una metodología coherente con todo } \\
\text { esto. La metodología en el aula depende del modelo de escuela que deseamos } \\
\text { construir. En nuestro caso lo estamos consiguiendo con los proyectos de inves- } \\
\text { tigación..." (Metodología) }\end{array}$ \\
\hline
\end{tabular}

En el centro 1 no vienen reflejados en el PEC los responsables de la actuación y desarrollo del proyecto. En el centro 2 se establece la coordinación del proyecto de ApS a través de la coordinación de metodología de ApS. Aspecto que conlleva la relación con los docentes que implementan el plan de centro relacionado con el ApS. En el centro 3 son todos los docentes los responsables ya que forma parte del PEC como proyecto global de centro (véase Tabla 6). 
Tabla 6. Resultados en la categoría sobre los responsables de la actuación

\begin{tabular}{|l|l|}
\hline CENTRO 1 & $\begin{array}{l}\text { No se encuentran referencias específicas a los responsables en el ámbito del } \\
\text { proyecto de comunidad de aprendizaje }\end{array}$ \\
\hline & $\begin{array}{l}\text { Los responsables de ApS se encuentran recogidos en: } \\
\text { “...El profesorado deberá, una vez aprobado en Consejo Escolar, ate- } \\
\text { nerse a cuanto hay escrito en el mismo, no pudiendo individualizar su } \\
\text { contenido, desconectándose del resto de compañeros..." (Marco legis- } \\
\text { lativo) } \\
\text { “...Coordinadora de Plan de Expresión Oral y Escrita: Desarrolla todo lo } \\
\text { referente al Plan de Expresión Oral y Escrita y al Plan de LEO-TIC..." (Coor- } \\
\text { dinación) } \\
\text { “...Coordinación de metodología ApS. y todos los proyectos relacionados } \\
\text { CENTRO } 2 \\
\text { proyectos de Aprendizaje Servicio que se realicen a nivel de centro. Así } \\
\text { como realizar las evaluaciones y memorias pertinentes..." (Coordinación } \\
\text { de comunicación) }\end{array}$ \\
\hline CENTRO 3 & Todos los docentes del centro \\
\hline
\end{tabular}

En los documentos de centro analizados no se muestran los resultados alcanzados tras la aplicación de los respectivos proyectos de innovación. En cuanto a los procesos de evaluación, el centro 1 no les señala, mientras que los centros 2 y 3 muestran cómo han de desarrollarse. En concreto, se establecen las fases y procesos que han de cumplir todos los docentes implicados, en el centro 2 y las que ha de cumplir toda la comunidad educativa, en el centro 3 (véase Tabla 7).

Tabla 7. Resultados en la categoría sobre los resultados alcanzados

\begin{tabular}{|l|l|}
\hline CENTRO 1 & $\begin{array}{l}\text { No se encuentran referencias específicas a las evaluaciones en el ámbito del } \\
\text { proyecto de comunidad de aprendizaje }\end{array}$ \\
\hline & $\begin{array}{l}\text { Dentro de los planes de centro en el relativo a ApS se encuentran los proce- } \\
\text { sos de evaluación: } \\
\text { “...Evaluación con el grupo: } \\
\text { Balance de los resultados del servicio } \\
\text { Reflexión y balance final de los aprendizajes } \\
\text { Proyección y perspectiva de futuro } \\
\text { Celebración } \\
\text { Evaluación del educador: } \\
\text { Evaluación del grupo y de cada alumno } \\
\text { Evaluación del trabajo en red con las entidades } \\
\text { Evaluación de la experiencia como proyecto de ApS } \\
\text { Autoevaluación del educador..." (Planes de centro) }\end{array}$ \\
& $\begin{array}{l}\text { Dentro de las fases que componen el desarrollo del Proyecto Roma, la asam- } \\
\text { blea final es la que se lleva a cabo en la fase de evaluación: } \\
\text { “...Asamblea Final. Es el momento de evaluar el proceso de trabajo realiza- } \\
\text { do por cada uno de los grupos y proponer nuevos proyectos: }\end{array}$ \\
\hline
\end{tabular}




\begin{tabular}{|l|l|}
\hline Presentación del proyecto de investigación en asamblea a los otros gru- \\
pos. Cada portavoz cuenta qué planificaron, qué dificultades han tenido \\
en el proceso y cómo las han resuelto, qué respuestas han encontrado a las \\
dudas de la asamblea y propone el mapa de aprendizajes que han elabo- \\
rado como síntesis de sus aprendizajes. También exponen sus dificultades \\
y limitaciones. \\
Evaluación de los aprendizajes genéricos y de los aprendizajes específicos. \\
Cuando todos los grupos han terminado de exponer y debatir sus proyectos, \\
se realiza el mapa de aprendizajes de la clase donde tienen que estar refle- \\
jados todos los aprendizajes de todos los grupos. Este mapa único y común \\
es el que cada niña y cada niño tiene en el libro que van construyendo a lo \\
largo del curso. El aprendizaje está concatenado, se une un proyecto con \\
otro, en una espiral sin fin. El conocimiento no acaba, siempre está unido \\
con algo siguiente. Normalmente los aprendizajes que se han producido \\
suelen ser diferentes a aquellos que deseaban aprender y que plantearon en \\
la asamblea inicial. Puede que se adquieran otros y algunos de los previstos \\
no se consigan. \\
Nuevo proyecto de investigación. Con los nuevos interrogantes surgidos en \\
los grupos y en la asamblea final planificamos un nuevo proyecto de inves- \\
tigación y de este modo seguimos investigando y aprendiendo. La base de \\
nuestro aprendizaje es la investigación. Sin investigación no hay aprendiza- \\
je..." (Metodología)
\end{tabular}

A tenor de los resultados analizados, se establecen diferencias entre los centros a la hora de desarrollar e implementar acciones encaminadas a la actualización pedagógica. Los centros en los que forma parte de su identidad, como el Centro 3, la acción vertebra todas las actuaciones, la organización y la planificación de actividades, lo que denota coherencia en el proceso. Por otro lado, los centros en los que no supera la implantación dentro de la organización del centro, como el caso del centro 1 , supone que las actuaciones se circunscriben a la acción en el aula, dejando de lado el potencial innovador que supone este tipo de acciones. De esta forma podemos observar como la autonomía pedagógica de centro da cabida a la inclusión y desarrollo de competencias que den satisfacción a las demandas actuales de la sociedad, a través de desarrollo del Proyecto Educativo de Centro entorno a esta finalidad, dando coherencia en las actuaciones.

\section{Discusión}

La confluencia de diferentes cambios de naturaleza política y socioeconómica ha provocado que en estos últimos años la política educativa transnacional haya diseñado un nuevo horizonte de sentido en la configuración competencial de una nueva ciudadanía, expresando de forma reiterada la demanda de reformas en los procesos educativos conducentes a su implementación.

En este contexto, la competencia global Changemaker emerge como elemento nuclear en la medida que asume intencionalmente la generación de habilidades, 
capacidades y actitudes para acometer el reto de desenvolverse en una sociedad híbrida, cambiante y con una alta volatilidad de conocimientos y valores.

La revisión teórica y el análisis cualitativo y categorizado de tres proyectos educativos de centro demuestran que el desafío de la implementación de la competencia global no comporta obligatoriamente modificaciones del marco jurídico del sistema educativo, ya que con la legislación actual se puede desarrollar esta competencia a través del descubrimiento del potencial innovador que genera la autonomía pedagógica de los centros educativos. La autonomía de centro se encuentra positivamente asociada con la implementación de la competencia global.

No obstante, la relación positiva evidenciada no se produce de modo automático, responde, por el contrario, a rasgos diferenciales. Así, en el centro 1 se produce una discrepancia entre lo que se desarrolla en el ámbito del aula, la implantación de un proyecto de comunidad de aprendizaje y lo recogido en el PEC, ya que no hay referencia explícita al mismo. Esta situación no excluye, como es obvio, el desarrollo del proyecto en el ámbito del aula, sin embargo, supone una anomalía. Por lo que se refiere a los centros 2 y 3 se recoge de forma explícita la inclusión de los proyectos de innovación en el procedimiento de actuación del centro, aunque no de igual forma. En el centro 2 su inclusión se centra en los planes de centro, mientras que en el centro 3 forma parte integral del PEC, justificándolo y dotándole de una configuración que satisface su inclusión en la metodología del Proyecto Roma. En ambos casos la autonomía de centro ha permitido configurar los procesos de aprendizaje y la adquisición de competencias de forma adaptada a la realidad social y a su entorno.

Los resultados de este trabajo aportan datos y argumentos que, además de responder a las demandas de los organismos internacionales proporcionando criterios de racionalidad para la decisión de la política educativa, también comportan en nuestros círculos pedagógicos la necesidad de impulsar una política curricular muy atenta a favorecer la autonomía de centro.

\section{REFERENCIAS BIBLIOGRÁFICAS}

Amadio, M., Opertti, R. y Tedesco, J. C. (2014). Un currículo para el siglo XXI: Desafíos, tensiones y cuestiones abiertas. Investigación y Prospectiva en Educación. (Documentos de Trabajo ERF, No. 9). Paris: UNESCO.

Azqueta Díaz de Aldaá, A. (2019). Análisis del concepto 'emprendedor' y su incorporación al ámbito educativo. Teoría de la Educación. Revista Interuniversitaria, 31(1), 57-80. https://doi.org/10.14201/teri.19756.

Bravo Monge, C. (2016). Las escuelas de pensamiento del emprendimiento social. Tec Empresarial, 10(3), 19-28. http://doi.org/10.18845/te.v10i3.2937.

Calleja, T., Collado, S., Macías, G. y San José, C. (2012). Educación en España. Motivos para la esperanza. Madrid: Mckinsey \& Company.

Comisión Europea (2010). Europa 2020. Una estrategia para un crecimiento inteligente, sostenible e integrador. COM (2010) 2020 final. 
Comisión Europea (2012). Un nuevo concepto de educación: invertir en las competencias para lograr mejores resultados socioeconómicos. COM (2012) 669 final.

Comisión Europea (2018). Recomendación del consejo relativa a la promoción de los valores comunes, la educación inclusiva y la dimensión europea de la enseñanza. SWD 13 final.

Consejo Europeo (2018). Recomendación del Consejo relativo a las competencias clave para el aprendizaje permanente. 2018/C 189/01.

Fernández-Laviada, A. (2016). Informe GEM España 2015. Madrid: CISE Centro Internacional Santander Emprendimiento.

Locatelli, R. (2018). La educación como bien público y común: Reformular la gobernanza de la educación en un contexto cambiante. Investigación y prospectiva en educación. (Documentos de trabajo, № 22). París: UNESCO.

Mella, O. (2003). Metodología cualitativa en ciencias sociales y educación: orientaciones teórico-metodológicas y técnicas de investigación. Santiago: Primus.

Montanero Fernández, M. (2019). Métodos pedagógicos emergentes para un nuevo siglo ¿Qué hay realmente de innovación? Teoría de la Educación. Revista Interuniversitaria, 31(1), 5-34. https://doi.org/10.14201/teri.19756.

OECD (2018). Marco de Competencia Global. Estudio PISA: preparar a nuestros jóvenes para un mundo inclusivo y sostenible. Madrid: Ministerio de Educación, Cultura y Deporte.

OECD (2019a). Estrategia de Competencias de la OCDE 2019: Competencias para construir un futuro mejor. Madrid: OECD Publishing, Paris/Fundación Santillana. https://doi.org/10.1787/e3527cfb-es.

OECD (2019b). Education Policy Outlook 2019: Working Together to Help Students Achieve their Potential. Paris: OECD Publishing. https://doi.org/10.1787/2b8ad56een.

Sancho, M.A. (2014). Posición de las comunidades autónomas ante la autonomía escolar de los centros públicos. Revista de Educación, 366, 64-86. https://doi. org/10.4438/1988-592X-RE-2014-366-276.

Schleicher, A. (2018). World Class: How to Build a 21st Century School School, Strong Performers and Successful Reformers in Education, Paris: OECD Publishing. https://doi.org/10.1787/9789264300002-en.

Scott, C. L. (2015). El futuro del aprendizaje (i) ¿Por qué debe cambiar el contenido y los métodos de aprendizaje en el siglo XXI? Investigación y Prospectiva en Educación. (Documentos de Trabajo ERF, No. 13). Paris: UNESCO.

Strauss, A. y Corbin, J. (2002). Bases de la investigación cualitativa. Técnicas y procedimientos para desarrollar la teoría fundamentada. Antioquía: Universidad de Antioquía. 
Tawil, S. (2013). La educación para la "ciudadanía mundial": marco para el debate. Investigación y Prospectiva en Educación. (Documentos de Trabajo ERF, No. 7). París: UNESCO.

UNESCO (2009). Comunicado - Conferencia Mundial sobre Educación Superior 2009: La nueva dinámica de la educación superior y la investigación para el cambio social y el desarrollo. París: UNESCO.

UNESCO (2015a). Educación 2030: Declaración de Incheon y Marco de Acción. París: UNESCO.

UNESCO (2015b). Replantear la educación: ¿Hacia un bien común mundial? París: UNESCO.

UNESCO (2016). La educación al servicio de los pueblos y el planeta: creación de futuros sostenibles para todos. Informe de seguimiento de la educación en el mundo 2016. París: UNESCO.

Viennet, R. y Pont, B. (2017). Implementación de políticas educativas: revisión de la literatura y marco propuesto. (Documentos de trabajo de educación de la OCDE, 162). París: OECD Publishing. https://doi.org/10.1787/fc467a64-en. 